\title{
Investigating the relationship between personality characteristics, self-control, and general health among the students of public and clinical psychology in Islamic Azad University of Ilam
}

Seyed Rahmatollah Mousavi Moghadam ${ }^{1}$, Somayeh Malekian ${ }^{2 *}$, Maryam Karamshahi

1. Department of Islamic Theology, Faculty of Medicine, Ilam University of Medical Sciences, Ilam, Iran

2. Department of Psychology, Research and Sciences Branch, Islamic Azad University, Ilam, Iran

*Corresponding author: Tel: +98 9169458608 Fax: +98

Address: Department of Psychology, Research and Sciences Branch, Islamic Azad University, Ilam, Iran

E-mail: Somaye1988.m@gmail.com

Received; 2015/04/22 revised; 2015/07/27 accepted; 2015/12/2

\begin{abstract}
Introduction: Mental health is a branch of science focused on promoting welfare, social welfare, and life health which is related to all periods (from birth to death) and aspects of life (family environment, school, university, appointment, and society). Personality is a collection of mental characteristics that consistently exists within individuals and influences their behaviors and thoughts. Self-control as one of the unique personality characteristics varies from one person to another.
\end{abstract}

Materials and methods: The present study is a correlational descriptive study. The target population of this research includes all graduate students of public and clinical psychology studying in Islamic Azad University of Ilam during academic year of 93-94. According to statistics there were approximately 536 students in this field. Out of this number 224 students were selected through simple random sampling. Measurement instruments included: Tangney and Baumeister (2004) Self-Control Scale, NEO Five-Factor Inventory, and Goldberg General Health Questionnaire. For the analysis of findings of this study KolmogorovSmirnov test, correlation, and regression analysis were run through statistical software of SPSS.

Results: Results of this research showed that personal characteristics and self-control predicted $86 \%$ of variance; that is, significantly predicted the level of mental health among students. Results of correlation revealed significant relationship between self-control and mental health. In addition, there were significant relation between personality traits and selfcontrol. In other words, it can be said that personality characteristics have a substantial role in predicting mental health and there is also significant relationship between the variables under study.

Conclusion: It can be said that self-control is one of the most important personality characteristics that influences individuals' mental health.

Keywords: Personality characteristics, Self-control, General health

\section{Introduction}

In order to determine factors effective in human health, researchers have made great effort. Meanwhile, health concepts have witnessed changes from day to day. Health involves accurate function of individual's body and even of mental aspects (1). Mental health allows students to use potential capabilities of their minds to achieve mental preparation. People who enjoy good mental hygiene (mental health) can attain mental calmness too. There is no precise definition for mental calmness, but it can generally be defined as individuals' general satisfaction from themselves and 
their environment which is characterized by lack of anxiety, depression, and apprehension (2). Mental health is a science focused on promoting welfare, social welfare, and life health which is related to all periods (pre-birth to death) and aspects of life (family environments, school, university, appointment, and society). Besides physiological needs, human beings have emotional, moral, religious, instructional, and directional needs and healthy life is produced through fulfilling these needs and maintaining balance between them (3). Personality is a collection of mental characteristics that consistently exists in individuals and influences their behaviors and thoughts. In other words, individuals' personality is a combination of mental characteristics that are used for determining the position of that individual in classifications (4). Due to the presence of numerous attributes and characteristics belonging to the topic of personality, personality psychologists have made efforts to categorize and organize this concept in order to systematize their knowledge in this field and, consequently, present systematic and coherent research studies. Such agreed-upon classifications have existed for so long in sciences like biology. Fortunately, despite facing such difficulties, it can be claimed that, after decades of research, personality psychologists are coming to a general agreement on the classification of human beings' personality characteristics, namely five factor model or big five personality factors (5). Self-control is one of the unique personality characteristics that varies from one person to another. That is, individuals with high levels of self-control depending on the extent of control they employ show different reactions and behaviors. In order to use this characteristic effectively, it should be nurtured because controlling excitements in social interactions is a very difficult task although researchers believe that public culture of a society effects the way individuals control themselves. Cultural psychology has confirmed that individuals are usually different with respect to their behaviors and through their particular behaviors are distinguished from each other; for instance, in most societies it is believed that only persons can have a personality who has stable, united and coherent characteristics and during time their personality remains unchanged and resists influence. Research has indicated that power of "self-control" in different periods of life ensures success. Agreement and conscientious are aspects of selfcontrol because these factors respectively ensure the ability to "adapt" one's behavior to other's feelings and wishes and tolerate desires (7). Shiri et al. (2014) in a study under the title "investigating the relationship between self-control and mental health: the case of Ilam university students" came to the conclusion that there is a significant relationship between selfcontrol and mental health. Further, results of this study indicated that there is a positively significant relationship between self-control and health dimensions (mental, social, physical) (8). Bahrami Khundabi (2003) in his study under the title "investigating the relationship between self-control and life quality among university students" concluded that there is a relationship between self-control and life quality of students; that is, as the level of self-control increases life quality also increases and consequently individuals' mental health promotes (9). Still in another study, Mohammadi Masiri et al. (2013) revealed that there is a negatively significant relationship between self-control and lack of mental health. Generally, it can be concluded that the variable of self-control plays an important role in individuals' mental health. Research has shown that self-control as one of social skills, can determine the level of success in one's life. Self-control has a positively significant relationship with mental health and interpersonal relationships (10). Dastourani and Pouryousef (2012) in their study came to 
the conclusion that mental health of staff members influences their personality characteristics. All personality characteristics including extroversion, flexibility, and conscientious except for delightfulness influence job satisfaction among staff members which in turn has direct impact on individuals' mental health (11). Break et al. (2005) conducted a study under the title "self-control as a modifier of the relationship between big five personality factors and interpersonal performance". Findings obtained from a sample of 102 students of executive management showed that high level of self-control lead to poor relations between three characteristics of five personality factors (e.g. extroversion, emotional stability, and openness of mind) in the experience and interpersonal performance of attendants. These results were repeated with the group of coevals and as it was expected self-control didn't modify the relationship between personality characteristics and the performance of the attendants group (12).

The purpose of this study was investigating the relationship between personality characteristics, self-control, and general health among the students of clinical and general psychology studying in Islamic Azad University of Ilam.

\section{Materials and methods}

The present research is a correlational descriptive study. The target population of this research consists of all graduate students of public and clinical psychology studying in Islamic Azad University of Ilam during academic year of 2014-2015. According to statistics there were approximately 536 students in this field. Sampling was carried out through simple random method. Sample size was calculated through Cochran formula, thereby 224 students were selected. Instruments utilized in this study include: Tangney and Baumeister Self-Control Scale (2004): the short version of this instrument consists of 13 items which is scored based on a five point likert scale. The range of individuals' scores was 36180 . In order to determine the reliability of this scale, two studies were conducted among graduate students. Cronbach alpha coefficient in both studies was $86 \%$ (13). General health questionnaire GHQ-28: the short version of this questionnaire including 28 items has been verified by academic organization of Iran, health sciences faculty under the supervision of Montazeri et al. Results of conducted studies show that this questionnaire has been administered among 748 students aged 18-25. Then, according to obtained results, reliability of this questionnaire was determined. Internal consistency reliability was assessed by computing Cronbach alpha coefficients which resulted in $\mathrm{r}=$ $87 \%$. Validity of this instrument was obtained through convergence with life quality questionnaire. In addition, according to factor analysis, two factors were identified, namely mental agitation and social malfeasance. Therefore, considering the verification of this instrument in Iran, it may have the capability of fast designation. Neo Five Factor Inventory (NEO-FFI): this questionnaire has a short version named (NEO-FFI) including 60 items which is used for evaluating 5 main factors of personality. This questionnaire is useful when there is time limitation and general information is needed. Furthermore, administration of this questionnaire is not costly and its scales enjoy high reliability and there is high correlation between these scales. Most importantly, despite other personality tests, there is little objection against this test (1). Items are answered based on a five point likert format scale (strongly disagrees, disagree, neutral, agree, and strongly agree). In short version of this instrument (NEO-FFI) scoring method is not the same for all materials. That is, for some items, 4 belongs to strongly disagree, 3 belongs to disagree, 2 belongs to neutral, 1 belongs to agree, and 0 belongs to strongly disagree. While for 
some other items it is vice versa (14). McCrae and Costa administered the questionnaire among 208 American students during an interval of three month and reliability coefficients were between 0.83 and 0.75 . Long term reliability of this questionnaire was also evaluated. In the span of 6 years a longitudinal study was conducted on the scales of khoei neuroticism, extroversion and openness to experience; as a result, reliability coefficients of 0.68 to 0.83 were revealed in personal reports and couples report. In an interval of 2 years reliability coefficients of adaptability and conscientious factors were 0.79 and 0.63 , respectively. For the analysis of findings obtained from this study KolmogorovSmirnov test, correlation, and regression analysis were run through statistical software of SPSS.

\section{Results}

Findings of the study are presented in Tables 1-3. According to the findings presented in Table 1, 14.7 percent of respondents had less than 25 years of age, 42.9 percent were between the ages of 25 $30,33.9$ percent were between the ages of $30-35$, and 8.5 percent of them were between the ages of 35-40 years old.
Table 1. Distribution of age and gender among the participants of the study.

\begin{tabular}{lccc}
\hline Variable & & Frequency & Percent $(\%)$ \\
\hline \multirow{4}{*}{ Age } & $<25$ years & 33 & 14.7 \\
& 30-25 years & 96 & 42.9 \\
& 35-30 years & 76 & 33.9 \\
& 40-35 years & 19 & 8.5 \\
\hline \multirow{2}{*}{ Gender } & Male & 159 & 71 \\
& Female & 65 & 29 \\
\hline
\end{tabular}

Results indicated that the age of the majority of respondents was between the ages of 25-30. Based on the findings given, 71 percent of respondents were male and 29 percent were female: that is, most of the respondents were male. As shown in Table 3 , correlation coefficient between personality characteristics and self-control was 0.568 which is statistically significant $(\mathrm{P}=0.001)$. Thus, the research hypothesis is accepted with 95\% confidence. Therefore, there is a positively significant relationship between two variables of personality characteristics and self-control. Further, data presented in Table 3 show that correlation coefficient between personality characteristics and self-control was 0.535 which also is statistically significant ( $\mathrm{P}=0.001)$. Thus, the research hypothesis is accepted with 95\% confidence implying that there is a positively significant relationship between self-control and mental health.

Table 2. Results of Kolmogorov-Smirnov test for mental health, personality characteristics, and self-control among participants.

\begin{tabular}{|c|c|c|c|c|c|}
\hline Variables & \multicolumn{2}{|r|}{$\mathbf{N}$} & \multicolumn{2}{|c|}{ Kolmogorov-Smirnov test } & P value \\
\hline Mental health & \multicolumn{2}{|r|}{224} & \multicolumn{2}{|c|}{5.588} & 0.000 \\
\hline Personality characteristics & \multicolumn{2}{|r|}{224} & \multicolumn{2}{|c|}{5.041} & 0.000 \\
\hline Self-control & \multicolumn{2}{|r|}{224} & \multicolumn{2}{|c|}{4.609} & 0.000 \\
\hline Variables & $\mathbf{B}$ & Standard error of B & Beta & $\mathbf{T}$ & P value \\
\hline Constant value & -18.849 & 19.460 & & -9.345 & 0.000 \\
\hline Personality characteristics & 1.632 & 0.183 & 0.570 & 8.942 & 0.000 \\
\hline Self-control & 0.858 & 0.145 & 0.377 & 5.908 & 0.000 \\
\hline
\end{tabular}

Table 3. Spearman's correlation coefficients for the association of personality characteristics and self-control parameters with self-control.

\begin{tabular}{lcc}
\hline & Spearman's correlation coefficient for association with self-control & P value \\
\hline Personality characteristics & 0.568 & 0.01 \\
Mental health & 0.535 & 0.01 \\
\hline
\end{tabular}




\section{Discussion}

Considering the output of Table 2 $(\mathrm{P}<0.05)$, the null hypothesis $(\mathrm{H} 0)$ is rejected and $\mathrm{H} 1$ which implies the nonnormality of data is accepted. Therefore, data presented in this table are not normal. Based on data analyses, the regression coefficient was 0.92, determination coefficient was 0.86, and adjusted determination coefficient was 0.86. The significance of regression was calculated through $\mathrm{F}=842.918$ which was statistically significant $(\mathrm{P}=0.001)$. Thus, considering the determination coefficient, it can be said that personality characteristics and selfcontrol significantly predicted students' mental health $(86 \%)$. In the Table 2 standardized coefficients of beta for predicting variables in the model fluctuate from 0.377 to 0.570 the lowest value of which belonged to self-control (0.304) and the highest value belonged to personality characteristics (0.570). According to the findings there is a relationship between personality characteristics, self-control, and general health among students of general and clinical psychology studying in Islamic Azad University of Ilam. The relationship between self-control and general health was stronger. Hypothesis 1 shows to what extent personality

\section{References}

1. Garousifarshi M. New approach in personality assessment (use of factor analysis in the study of personality). Printing. Tabriz: Publication of Daniel and the research community, 2002.

2. Mohammadi D. MehrabizadehHonarmand M. Relationship between religious attitude and position control and the role of gender. J Psychol Res. 2014; 9(3 and 4): 47-62.

3. Lashkari M. Mental health in Islam. J Qazvin Uni Med Sci. 2015; 24(2):5617.

4. Rabinz SP, Debi SD. Organizational Behavior. 4th ed.1998. Translation by Amrabi M, Hamid Rafiei M, characteristics and self-control predict students' mental health. According to determination coefficient, personality characteristics and self-control significantly predicted students' mental health $(86 \%)$. Results of this study are in line with the results obtained by Bahrami Khundabi (2003) (9). Hypothesis 2 states that there is a significant relationship between self-control and mental health. Based on these findings this hypothesis is also accepted which supports the findings of previous research conducted by Shiri et al. (2013) (8), Mohammadi et al. (2012) (10), and Dastourani and Pour Yousef (2012) (11).

\section{Conclusion}

Previous research confirms the effect of self-control on individuals' mental health. Because a person who is not able to express his/her interpersonal oppositions when making personal decisions and cannot control him/herself in difficulties would easily be involved in unimportant issues. Thus, it can be said that self-control is one of the most important personality characteristics that influences mental health.

Asrariershad B. Tehran Publications Office of Cultural Research: 2007.

5. John OP, Srivastava S. The Big Five trait taxonomy: History, measurement, and theoretical perspectives. In: L. A. Pervin, \& O. P. John (Eds.), Handbook of personality: Theory and research; New York: Guilford Press.1999.P. 102-38.

6. Markis HR. Culture and personality. Brief for an arranged marriage. J Res Person. 2004; 38:75-83.

7. Desmond SA. Religion, prosocial learning, self-control, and delinquency. J Person. 2008;4(1):541-7. 
8. ShirI A. Bagheritadi N, Mehrpouyan $\mathrm{N}$, Heydarianghaleh $\mathrm{H}$. Examine the relationship between self-control and mental health: A Case Study of ILAM University. J Manag Entrepreneurship Econom Develop. 2014;8(2):682-8.

9. Bahramikhondabi F. The relationship between self-control and quality of life among male and female university students. J Ment Health. 2003;5(3):976-81.

10. Mohammadimosiri F. The role of selfcontrol, quality of relationships with parents and school mental health and anti-social behavior in adolescents. Develop Psychol J. 2012; 8(32):398-7.

11. Dastourati M, Pourusef S. Investigate the relationship between personality traits and mental health and job satisfaction. Inspections. 2012; 4(20):110-5.
12. Barrick MR, Parks LM, Michael k. Self-Monitoring as a moderator of the relationships between personality traits and performance. Person Psychol. 2005; 58(4):745-65.

13. Tangeny JP, Baumeister RF. High selfcontrol predictts good adjustment, less pathology, better grades, and interpersonal success. J Person. 2004; 72(2):271-322.

14. Samari AA. The study of the interrelationships of personality traits and job stress in the workplace. J Ment Health. 2007; 5(21 and 22):19-28.

15. Baron RA. Personality and organizational conflict: Effect of the type a behavior pattern and selfmonitoring. Organ Behav Human Decision Proc. 1989; 44(2):281-96. 